\title{
Development of an ionic-liquid-based dispersive liquid-liquid microextraction method for the determination of antichagasic drugs in human breast milk. Optimization by central composite design
}

Juan M. Padró ${ }^{1}$, Rocío B. Pellegrino Vidal ${ }^{1}$, Romina N. Echevarria ${ }^{1}$, Alicia N. Califano ${ }^{2}$, Mario R. $\operatorname{Reta}^{1, *}$

${ }^{1}$ Laboratorio de Investigación y Desarrollo en Métodos Analíticos (LIDMA), Facultad de Ciencias Exactas, Universidad Nacional de La Plata (UNLP), Calle 49 y 115 (1900) La Plata, Bs. As., Argentina.

${ }^{2}$ Centro de Investigación y Desarrollo en Criotecnología de Alimentos (CIDCA), CONICET, Facultad de Ciencias Exactas, Universidad Nacional de La Plata (UNLP), Calle 47 y 116 (1900) La Plata, Bs. As., Argentina.

"Corresponding author e-mail: mreta@quimica.unlp.edu.ar

Keywords: antichagasic drugs, central composite design, dispersive liquid-liquid microextraction, ionic liquids, liquid chromatography. 


\section{Abstract}

Chagas disease constitutes a major public health problem in Latin America. Human breast milk is a biological sample of great importance for the analysis of therapeutic drugs, as unwanted exposure through breast milk could result in pharmacological effects in the nursing infant. Thus, the goal of breast milk drug analysis is to inquire to which extent a neonate may be exposed to a drug during lactation.

In this work, we developed an analytical technique to quantify benznidazole and nifurtimox (the two antichagasic drugs currently available for the medical treatment) in human breast milk, with a simple sample pre-treatment followed by an ionic-liquid-based dispersive liquid-liquid microextraction combined with high-performance liquid chromatography and UV detection. For this technique, the ionic liquid 1-octyl-3-methylimidazolium hexafluorophosphate has been used as the "extraction solvent". A central composite design was used to find the optimum values for the significant variables affecting the extraction process: volume of ionic liquid, volume of dispersant solvent, ionic strength, and $\mathrm{pH}$. At the optimum working conditions, the average recoveries were 77.5 and $89.7 \%$, the limits of detection were 0.06 and $0.09 \mu \mathrm{gL}^{-1}$ and the inter-day reproducibilities were 6.25 and $5.77 \%$ for benznidazole and nifurtimox, respectively. The proposed methodology can be considered sensitive, simple, robust, accurate, and green.

\section{$1 \quad$ Introduction}

Chagas disease is endemic in Central and South America. Approximately 16-18 million people are infected with Trypanosoma cruzi (T. cruzi) and 100 million are at risk of infection [1-5]. Two drugs are available for the etiological treatment of Chagas disease: nifurtimox (NFX) and benznidazole (BNZ) [6,7]. Currently, NFX and BNZ are used in Argentina, Brazil and Uruguay to treat the acute or indeterminate chronic phases of Chagas disease $[1,4,8,9]$. These drugs are widely 
accepted for the treatment of the acute phase of the disease $[2,4,7]$. However, their use during the chronic phase remains controversial $[4,7,10]$.

The congenital route of infection might be relevant to endemic as well as non-endemic areas in future years after migration of infected individuals. Public health policies have been implemented in endemic areas with the aims of identifying pregnant women positive for $T$. cruzi, as well as promptly treating infected children immediately after parasitological and serological confirmation $[1,2,8]$. It is important to emphasize that the duration of both NFX and BNZ chemotherapeutic effects, as well as its toxicity, are fundamentally controlled by its reductive metabolism in many organs and the feces [11-13].

Human breast milk is a biological sample of great importance for the analysis of therapeutic drugs, as unwanted exposure through breast milk could result in pharmacologic effects in the nursing infant. Human breast milk is characterized by a high content of proteins, lipids, and carbohydrates which may be vary significantly in composition and concentration [14] depending on several factors including stage of lactation, breastfeeding routine, age, diet, and others [15]. These variables can affect the recovery of an extracted drug, the analytical method precision, the accuracy, and the sample compatibility with a subsequent chromatographic technique [14].

Drug transfer from plasma into breast milk occurs primarily by passive diffusion depending on molecular weight, drug ionization, lipid solubility, protein binding, and mammary blood flow [1619]. However, it remains unknown to what extent NFX or BNZ are transported into breast milk. Without this knowledge, most physicians who treat adult patients with Chagas disease refuse to treat women during lactation.

Several reports have been published on detection methods for BNZ in biological fluids. The different procedures used in the literature have been detailed in our previous work [20]. Limits of detection between 0.04 and $0.14 \mu \mathrm{g} \mathrm{mL} L^{-1}$ and $\mathrm{RSD} \%$ between 1.3 and $10 \%$ were obtained, with recoveries from 70 to $97 \%$. Recently, Martínez et al. have published an analytical procedure to determine BNZ in urine by UHPLC-MS/MS with a simple sample pretreatment consisting of a LLE 
with dichloromethane [21]. The methodology is quick, reproducible and selective but, although is sensitive, the detection limit $\left(0.75 \mu \mathrm{g} \mathrm{m}^{-1}\right)$ resulted to be similar to others previously published in the literature due to a serious matrix effect (90\% of signal suppression) which affected the ESI-MS/MS detector. Up to our knowledge, the only published paper about determination of $\mathrm{BNZ}$ in human breast milk is that corresponding to Marsón et al. [22]. They developed a simple and fast RP-HPLC-UV method with a sample pretreatment that involves acid protein precipitation followed by tandem microfiltration. The procedure has proven to be robust, quite reproducible and sensitive with a LOD of $0.3 \mu \mathrm{g} \mathrm{mL} \mathrm{L}^{-1}$ and a recovery of $70 \%$.

In the case of NFX, a few methods also detailed in our previous work [20] have been published for its determination in biologic fluids. Quantitative determinations of the drug at a sensitivity of at least $0.5 \mathrm{mg} \mathrm{mL}^{-1}$ were reported [23]. These studies resulted in the development of an easy sample-preparation procedure for pharmacokinetic studies in patients with chronic renal failure [23]. However, no analytical methods have been specifically reported for NFX analysis in human breast milk.

Dispersive liquid-liquid microextraction (DLLME) is a novel sample preparation technique $[24,25]$ applied to the determination of several analytes in different matrices. It is based on a ternary solvent system in which the extraction solvent (e. g., dichloromethane, octanol or toluene) is dissolved in the miscible disperser solvent (e. g., methanol, acetonitrile or isopropanol) are rapidly injected into the liquid samples by using a syringe. The disperser solvent must be miscible also with the aqueous sample. At the beginning of the dispersion, exceedingly small droplets are formed that enable a maximal increase in mass transfer. These droplets then collapse to form the extractant phase containing the analytes in an extremely small volume, thus achieving high enrichment factors.

Room-temperature ionic liquids (RTILs), a form of melting salts composed of organic cations and either organic or inorganic anions, have emerged as possible environmental friendly solvents $[26,27]$ with the consequent wide applications in the separation sciences [28-31]. This is due to their unique properties, such as low volatility, chemical and thermal stability, and good solubilization 
properties toward both organic and inorganic compounds. Thus, RTILs are progressively replacing the typical organic solvents in LLE. The RTILs have been used as extractants in DLLME (ILDLLME) in several studies such as the determination of nonsteroidal anti-inflammatory drugs in urine by HPLC-UV [32], aryloxyphenoxy-propionate herbicides in aqueous samples by HPLC-DAD [33], insecticides by HPLC-DAD [34], sudan dyes and phthalate plasticizers in food (high-risk solid condiments and sauces) by HPLC-DAD [35], organophosphorus pesticides (OPPs) in apple and pear by HPLC-DAD [36] or polyaromatic hydrocarbons by HPLC-FLD [37] in water samples.

In a previous work, we have used a IL-DLLME combined with HPLC-UV for the determination of NFX and BNZ in human plasma [20]. This procedure yielded significant improvements over those previously reported in the literature and has several advantages, including high inter-day reproducibility (1.02\% and 3.66\% for nifurtimox and benznidazole, respectively) and extremely low detection limits (15.7 and $26.5 \mathrm{ng} \mathrm{mL}^{-1}$ for nifurtimox and benznidazole, respectively). The proposed methodology is inexpensive, simple, fast and requires small amount of sample, an advantage consistent with the practicability requirements in current clinical research or therapeutic monitoring.

Based on a theoretical analysis, transference of NFX into breast milk is expected to be limited [38], and unlikely to lead to a significant exposure of the breastfed infant. On the other hand, up to date there are no theoretical or experimental data about transference of BNZ into human breast milk. According to safety, scientific and regulatory reasons, the evaluation of the potential exposure of children to antichagasic drugs through breast milk in mandatory. Based on these facts, studies about the degree of transference of BNZ and NFX into breast milk are required. Thus, a suitable procedure for sample pretreatment with a subsequent analytical procedure needs to be developed for a reliable drug determination in that biological matrix characterized by its complexity and variability.

The use of multivariate experimental design techniques is becoming increasingly widespread in analytical chemistry and other sciences. Multivariate designs, which allow the simultaneous study 
of several control variables, are faster to implement and more cost-effective than traditional univariate approaches [39]. The central composite design (CCD) [40] is one of the most used multivariate techniques which combines a two-level full or fractional factorial design with at least one point at the center of the experimental region (usually, the starting value for each variable near to what is believed the optimum value is located), and additional points ("star points") selected to obtain properties such as rotatability or orthogonality to fit quadratic polynomials. Thus, CCD has been the most accepted experimental design for second-order models [41].

In this work, we applied IL-DLLME-HPLC-UV for the first time for the determination of BNZ and NFX in human breast milk. We optimized the critical experimental parameters controlling the extraction process (volume of ionic liquid, volume of disperser solvent, $\mathrm{pH}$ and concentration of potassium chloride) by using a multivariate approach known as $\mathrm{CCD}$, which allows linear and quadratic effects of the significant variables, as well as the interactions between them, to be estimated.

\section{$2 \quad$ Materials and methods}

\subsection{Chemicals and materials}

The RTIL 1-octyl-3-methylimidazolium hexafluorphosphate $\left(\left[\mathrm{C}_{8} \mathrm{C}_{1} \mathrm{im}\right]\left[\mathrm{PF}_{6}\right]\right)$ was synthesized in our laboratory with a procedure adapted from the literature [42]. Reagents were of analytical grade or better: benznidazole (Roche, Buenos Aires, Industria Argentina), nifurtimox (Bayer, Leverkusen, Germany), potassium hexafluorophosphate, $98.0 \%$ (Aldrich, WI, USA), 1-methylimidazole, $\geq 99.0 \%$ (Merck, Hohenbrunn, Germany), 1-bromoctane, $\geq 99.0 \%$ (Aldrich, WI, USA), sodium hydroxide (Analar, Poole, England), potassium chloride, sodium phosphate dibasic anhydrous (Anedra, Argentina), potassium phthalate monobasic, $\geq 99.5 \%$ (Fluka, Buchs, Germany), methanol HPLC grade (Baker's Analyzed, Phillipsburg, NJ, USA). Solutions were prepared with MilliQ ${ }^{\circledR}$ water. 
The 100 and $25 \mu \mathrm{L}$ microsyringes were supplied by Hamilton, (Reno, NV, USA) and Agilent Technologies (Australia), respectively. The micropipettes were purchased from Eppendorf (Hamburg, Germany).

\subsection{Instrumentation and chromatographic conditions}

An HP 1100 liquid chromatograph equipped with a binary pump, a thermostat-controlled column compartment, degasser and variable-wavelength detector connected to a Data Apex CSW workstation (Data Apex, Czech Republic) was used. Chromatographic analysis was performed on a 250 x $4.6 \mathrm{~mm}$ ID $(5 \mu \mathrm{m})$ Zorbax Eclipse XDB-C 18 column (Agilent). Methanol/water (45:55 v/v) was used in the mobile phase. The organic phase was prefiltered through a $0.22 \mu \mathrm{m}$ nylon membrane (Osmonics-Magna) and the aqueous phase was prefiltered through a $0.45 \mu \mathrm{m}$ cellulose-nitrate membrane (Micron Separations). The detector was set at $320 \mathrm{~nm}$ for BNZ and $395 \mathrm{~nm}$ for NFX, at which wavelengths the RTILs studied absorb no radiation. The injector (Rheodyne Model 7725i, Cotati, CA, USA) was fitted with a $5 \mu \mathrm{L}$ loop. The flow rate was set at $1 \mathrm{~mL} \mathrm{~min}^{-1}$.

An Eppendorf $5417 \mathrm{C} / \mathrm{R}$ centrifuge operating at $10000 \mathrm{rpm}$ for the optimization and quantification experiments were used. A Vortex Genie 2 (Scientific Industries, USA) mixer was used for mixing the aqueous and the ionic liquid phases. For the $\mathrm{pH}$ measurements, a combined glass Metrohm electrode in a commercial Accument AR $25 \mathrm{pH} / \mathrm{mV} / \mathrm{Ion} /$ Meter (Fisher Scientific) $\mathrm{pH}$ meter was used. Water was purified with a Milli-Q system (Millipore).

\subsection{Human breast milk samples}

Eight breast milk samples of different patients were obtained from lactating mothers with Chagas disease treated with BNZ or NFX (BNZ 5-10 $\mathrm{mg} \mathrm{kg}^{-1} \mathrm{~d}^{-1}$ every $12 \mathrm{~h}$ for 30 days, or NFX 10- 
$15 \mathrm{mg} \mathrm{kg}^{-1} \mathrm{~d}^{-1}$ every $8 \mathrm{~h}$ for 30 days), who were enrolled in a clinical study approved by both ethical and research review committees of Ciudad Autónoma de Buenos Aires Children's Hospital Dr. Ricardo Gutierrez, Buenos Aires City, Argentina. The patients were not taking abuse drugs or any other medication except BZN or NFX. The samples were taken from the patients between the fourth and the tenth day after the drugs were administered. Around $5 \mathrm{~mL}$ of breast milk per patient was collected in polypropylene flasks with lid, without additives. All samples were maintained in the freezer until use.

\subsection{Sample pretreatment}

Several types of mixtures, some of them taken from the literature [43], to eliminate the proteic and lipidic material were tested. The most efficient one consisted of a precipitation mixture of $\mathrm{HClO}_{4}$ $(0.15 \mathrm{M}) / \mathrm{H}_{3} \mathrm{PO}_{4}(0.15 \mathrm{M}) / \mathrm{MeOH}$. In a $2.0 \mathrm{~mL}$ polypropylene microcentrifuge tube containing $250 \mu \mathrm{L}$ of human breast milk free of the antichagasic drugs spiked with $5 \mu \mathrm{L}$ of BNZ $486 \mathrm{mg} \mathrm{L}^{-1}$ and NFX $574 \mathrm{mg} \mathrm{mL}^{-1}, 800 \mu \mathrm{L}$ of the precipitation mixture were added. Then all tubes were shook (high speed) during $2.0 \mathrm{~min}$ and incubated at $80^{\circ} \mathrm{C}$ for $60 \mathrm{~min}$. Next the samples were centrifuged at $10,000 \mathrm{rpm}$ for $20 \mathrm{~min}$ and the supernatant was separated from the solid material (lipids and proteins) with a Pasteur pipette. Then, $200 \mu \mathrm{L}$ of the precipitation mixture were added to the remaining residue, shaken and centrifuged, the obtained supernatant was added to the first extract and filtered using a micro-mate interchangeable syringe (Popper \& Sons, New Hyde Park, NY, USA) with $0.22 \mu \mathrm{m}$ cellulose nitrate membrane filters.

The resulting IL-DLLME procedure optimized by CCD was as follows: I) for NFX: $50 \mu \mathrm{L}$ of a $2 \mathrm{M} \mathrm{NaOH}$ solution and $150 \mu \mathrm{L}$ of $\mathrm{KCl}$ solution $30(\%, w / v)$ were added to the obtained supernatant. Then, $122 \mu \mathrm{L}$ of a mixture of $42 \mu \mathrm{L}$ of $\left[\mathrm{C}_{8} \mathrm{C}_{1} \mathrm{im}\right]\left[\mathrm{PF}_{6}\right]$ (the extractant solvent) with $80 \mu \mathrm{L}$ of $\mathrm{MeOH}$ (the dispersant solvent) were rapidly injected with a micro syringe and brought to $1.5 \mathrm{~mL}$ with MilliQ water. II) For BNZ, $100 \mu \mathrm{L}$ of $\mathrm{KCl}$ solution $30(\%, w / v)$ and $45 \mu \mathrm{L}$ of $2 \mathrm{M} \mathrm{NaOH}$ 
solution were added to the obtained supernatant. Finally, $143 \mu \mathrm{L}$ of a mixture of $42 \mu \mathrm{L}$ of $\left[\mathrm{C}_{8} \mathrm{C}_{1} \mathrm{im}\right]\left[\mathrm{PF}_{6}\right]$ with $101 \mu \mathrm{L}$ of $\mathrm{MeOH}$ were rapidly injected and brought to $1.5 \mathrm{~mL}$ with MilliQ water.

The obtained samples (I and II) are shaken in a vortex for $6.0 \mathrm{~min}$ and centrifuged at 10,000 rpm for $20.0 \mathrm{~min}$. The aqueous phase on top was removed with a Pasteur pipette. The obtained RTIL phase, measured five times by a calibrated microsyringe, was $40 \pm 5 \mu \mathrm{L}$. Finally, $5 \mu \mathrm{L}$ of the extractant was injected into the chromatographic column. All experiments were performed in triplicate.

\subsection{Experimental design and statistical analysis}

A four-factor $\mathrm{CCD}[44]$ for the $\mathrm{pH}$, volume of ionic liquid $\left(V_{\mathrm{IL}}\right)$, methanol volume $\left(V_{\mathrm{MeOH}}\right)$, and $\mathrm{KCl}$ concentration $\left(C_{\mathrm{KCl}}\right)$, with the center point replicated 12 times was used. Each of the eight axial and 12 factorial points were replicated twice, given a total of 60 individual experiments (runs).

Table 1 shows the actual and codified compositions. The runs were randomized to exclude block effects. The percent recovery of BNZ and NFX (R\% BNZ and R\% NFX, respectively) were analyzed in duplicate in each run.

A response surface methodology (RSM) was used to analyze the effect of the four independent variables on the responses using the second-order polynomial response surface with the corresponding interactions:

$$
Y=\beta_{0}+\sum_{i=1}^{4} \beta_{i} x_{i}+\sum_{i=1}^{4} \beta_{i i} x_{i}^{2}+\sum_{1<i<j}^{4} \beta_{i j} x_{i} x_{j}+\sum_{1<i<j<k}^{4} \beta_{i j k} x_{i} x_{j} x_{k}+\beta_{i j k m} x_{i} x_{j} x_{k} x_{m}+\varepsilon
$$

where $Y$ is the corresponding response variable; $\mathrm{x}_{\mathrm{i}}$ are the codified independent variables, $\beta_{0}$ the constant term, $\beta_{i}$ represents the coefficient of the linear parameters, $\beta_{i i}$ corresponds to the parameters of the quadratic terms, $\beta_{i j}, \beta_{i j k}$, and $\beta_{i j k m}$ are the coefficients of the interaction parameters, and $\varepsilon$ is the 
residual associated with the experiment. A stepwise methodology was followed to determine the significant terms in Eq. (1). Differences in the computed parameters were considered significant when the computed probabilities were less than $0.05(P<0.05)$.

All statistical analysis, generation of response surfaces, desirability functional analysis, optimization and 3D and contour plots were accomplished using the Expert Design (trial version 7.1.6, Stat-Ease, Minneapolis, USA) statistical software.

\subsection{Optimization and verification}

The optimum experimental conditions calculated using the predictive equations derived by RSM were employed for the microextractions of BNZ and NFX. Finally, the results were statistically compared to the values predicted by the mathematical model.

\section{$3 \quad$ Result and Discussion}

\subsection{Optimization of the IL-DLLME procedure by central composite design}

As discussed in the introduction, the CCD methodology has the advantage of reducing the number of trials allowing to find more accurately the optimal values of the different variables affecting the extraction performance and, simultaneously, to detect the presence of interactions between them. Therefore, using the optimal experimental parameters obtained in our previous work by using the "step-by-step" procedure for the determination of BNZ and NFX (Figure 1.a and 1.b, respectively) in human plasma [20] as the starting point, in this work we refined the optimization procedure to find the optimal parameters closer to the "real ones", by applying a CCD procedure. Since the amount of available sample was low, we scaled the obtained parameters to work in a microscale level. The final procedure is detailed in Section 2.4. 
The percent recovery, $\mathrm{R} \%$, was the response for which maximum values we pretend to obtain by changing each experimental factor. The type of extractant (RTIL), dispersant and type of salt were the same used in the "step-by-step" methodology previously mentioned [20]. Therefore the remaining factors, considered most influential on the responses, were included in the CCD: volume of RTIL, $V_{\mathrm{IL}}$; volume of dispersant, $V_{\mathrm{MeOH}}, \mathrm{pH}$, and saline effect, for which the variable in this case is the $\mathrm{KCl}$ concentration, $C_{\mathrm{KCl}}$. The levels chosen for those variables correspond to values that are close to the corresponding optimal values obtained by the "step-by-step" procedure.

\subsection{CCD experiments and responses}

Considering the previously mentioned four selected variables, a CCD consisting of a $2^{4}$ factorial design with six star points positioned at $\pm \alpha$ from the center of the experimental domain was carried out. The axial distance $\alpha$ was chosen with a value of 2.0 to fulfill the rotatability condition, i.e., the design generates information uniformly in all directions. The design was completed with six experiments at the central point, so that the number of degrees of freedom for the lack-of-fit equals that for replications. Therefore, the complete design consisted of 30 (i.e. $2^{4}+2 \times 4+6$ ) experiments.

The conditions of each experiment corresponding to the CCD procedure are listed in Table 1. The experiments and their responses are presented in Supporting Information, Table S1. They were performed in a random order to ensure the independence of the results by minimizing the effect of uncontrolled factors. The obtained design was repeated a week later to obtain a duplicate. Thus, a total of 60 experiments were performed. These trials were divided into three blocks performed on three consecutive days. As a comparison, the "step-by-step" design used in our previous work [20] for determination of the same antichagasic drugs in human plasma required a total of 237 experiments (including replicates).

The results obtained by the CCD were used to build a model based on linear-type interactions where each response was adjusted to a polynomial equation. In each model, the coefficients were calculated by multiple regressions using a backward elimination strategy. This strategy consisted of 
initially building a model and then evaluating each term through an analysis of variance (ANOVA) test to eliminate one by one the non-significant terms $(\alpha=0.05)$. The iteration stopped when all the remaining terms satisfied a desired probability level. The obtained model was finally validated by verifying that the same result was significant according to an ANOVA test with $P<0.05$ and that their lack of fit was not significant at $P>0.05$.

The response surfaces for BNZ and NFX are shown in Eq. (2) and (3).

$$
\begin{gathered}
R \%(\mathrm{BNZ})=44.61+1.15 V_{\mathrm{IL}}-3.01 \mathrm{pH}(2) \\
R \%(\mathrm{NFX})=116.82-0.87 V_{\mathrm{IL}}-27.66 C_{\mathrm{KCl}}+0.75 V_{\mathrm{IL}} C_{\mathrm{KCl}}(3)
\end{gathered}
$$

It can be observed that for BNZ the experimental variables affecting the extraction process are the volume of RTIL and $\mathrm{pH}$, while for NFX the significant terms are the volume of RTIL, the concentration of $\mathrm{KCl}$ and the interaction between them $\left(V_{\mathrm{IL}} C_{\mathrm{KCl}}\right)$. For both drugs, the volume of dispersant is not relevant in the final recovery. The extraction and centrifugation times were not considered in the CCD because adding one or two experimental factors increased significantly the number of experiments required. Therefore, for the extraction and centrifugation times we selected the optimal values obtained by the "step-by-step" procedure, i.e., 6.0 and $20.0 \mathrm{~min}$, respectively.

The next step was to find the factor conditions (volume of ionic liquid, volume of dispersant, concentration of $\mathrm{KCl}$ and $\mathrm{pH}$ ) that maximized the response. Figures 2.a and 2.b show the response surfaces for both analytes. For BNZ, the surface diagram shows that $R \%$ increases linearly with the volume of the RTIL (as expected since more analyte is extracted) and with decreasing pH. Since no $\mathrm{p} K_{\mathrm{a}}$ data is available for these antichagasic drugs, no conclusions can be extracted from this last result. For NFX it can be observed that $R \%$ increases linearly with the increasing volume of the RTIL and with decreasing $\mathrm{KCl}$ concentration. This latter behavior could be surprising if the "salting-out" effect is the only factor considered. However, it was previously observed that coulombic interactions between the ions of the LI and the added salt can be present [20]. This phenomenon increases the 
solubility of the RTIL into the aqueous phase with the consequent increasing of analyte solubility in this phase.

From a simple visual inspection of the response surfaces, it is not possible to find one optimal experimental condition, i.e., the best combination of levels of the four factors necessary to achieve simultaneously the maximum responses. Thus, to optimize the responses for each compound, the Desirability function, D, approach [45] was used. Each $\mathrm{D}_{\mathrm{i}}$ function takes values between 0 and 1 , where 0 corresponds to a completely undesirable value and 1 to a completely desirable one, i.e., an ideal response. The optimum values were obtained by using the corresponding fitted model and selecting the optimization criterion to be achieved for each compound. All factors were also included in the simultaneous optimization procedure to prioritize the use of certain more desirable conditions within the experimental region. In both cases the desirable ranges for the variables were selected and established whether it should be maximized, minimized, remain within a range or reach a target value. The approach used in this case corresponds to maximize $R \%$. The predicted conditions that maximized the recovery of the IL-DLLME are shown in Table 2. The overall $D$ function under these conditions was equal to 0.957 and 1.000 for the BNZ and NFX, respectively, which show that the optimum values obtained with the CCD model correctly predict the maximum percent recovery of the analytes. The predicted maximum recoveries were 78.0 and $91.87 \%$ for BNZ and NFX, respectively. In Section 3.3 these values are compared with the experimental ones.

It can be observed in Table 2 that the optimal values predicted by the CCD procedure are: $i$ ) for the volume of ionic liquid, somewhat higher than the ones determined by the step-by-step procedure; ii) for the dispersant volume (methanol), higher in the case of BNZ but lower in the case of $\mathrm{NFX}$; iii) for the $\mathrm{pH}$, lower in the case of BNZ and almost equal for NFX and; vi) for the $\mathrm{KCl}$ concentration, almost equal in the case of BNZ but higher in the case of NFX. These differences can be attributed to the fact that, unlike the empirical step-by-step procedure, the CCD is a statistical methodology, which can predict values for the different variables closer to the real ones and also, to 
the fact that the extraction experiments were performed on very different matrices (human breast milk vs. ultrapure water).

\subsection{Validation of the proposed IL-DLLME-HPLC-UV methodology}

Matrices can affect the analyte response by two different mechanisms: $i$ ) due to the matrix components present at the time of measuring, or ii) some components coming from the sample pretreatment, indispensable because of the matrix interferences. Any operation to which the sample is subjected before the measurement can result in a matrix effect. Thus, their effects must be studied.

\subsection{Matrix effect studies}

To study the matrix effects, different calibration curves (see Table 3) obtained by spiking with BNZ and NFX three different samples of human breast milk with no initial content of the drugs were constructed. Then, the slopes obtained by linear regression for the different samples were statistically compared by using a $t$-test at $5 \%$ probability $(P=0.05)$ [46]. It is observed in Table 4 that, in all instances, $t_{\text {calculated }}>t^{\prime}$ tabulated, that is there were significant differences between slopes using different breast milk samples, so matrix effects were therefore present. As a consequence, for the quantification of BNZ and NFX in human breast milk samples, the standard addition method was used for each milk sample.

\subsection{Figures of merit}

As an internal validation of the proposed IL-DLLME-HPLC-UV methodology, the following figures of merit were evaluated: accuracy, repeatability, reproducibility, LOD, lower and upper LOQ, the linear range (LR), enrichment factor (EF), and extraction recovery (R\%). 
The LOD was calculated by two procedures to compare with other studies in the literature: $\mathrm{S} / \mathrm{N}=3.0$ and the IUPAC definition of $\mathrm{LOD}=3.29 s_{0}$, where $s_{\mathrm{o}}$ is the SD of the blank [46]. The lower LOQ was evaluated as $\mathrm{S} / \mathrm{N}=10.0$ and by the IUPAC definition of $\mathrm{LOQ}=10 s_{\mathrm{o}}$ [46]. The upper LOQ was determined by the lack-of-fit procedure [46], i.e., by eliminating the highest value and applying the statistical test again with the remaining points. This process is repeated until the data can be adjusted to a straight line. The linear range (LR) is defined by the lower and the upper LOQs. LOD and LOQ values for both drugs are shown in Table 5.

To validate the accuracy and precision of the determinations, the samples of human breast milk (without initial content of the drugs) were spiked with each target drug at three different concentration levels within the linear range of the calibration curve. The precision was determined by the repeatability (intra-day) and reproducibility (inter-day determinations) with samples containing 5.0, 10.0, and $15.0 \mu \mathrm{g} \mathrm{mL}^{-1}$ of NFX and BNZ. Repeatability and reproducibility were expressed as the $\mathrm{RSD} \%$ with respect to measurements made in triplicate. The same drug concentrations were analyzed over three consecutive days to determine inter-day precision. The accuracy was determined by spiking the sample without analyte ("blank milk") with known amounts of the standards and calculating the percentage relative error by using the actual and found concentrations. The results are summarized in Table 6. The enrichment factors (EFs) and recoveries ( $R \%)$ were calculated by using Eq. (4) and (5), respectively.

$$
E F=\frac{C_{I L}}{C_{a q}}
$$

where $C_{\mathrm{IL}}$ and $C_{\mathrm{aq}}$ are the analyte concentrations in the IL phase and the initial aqueous solution, respectively 


$$
R \%=100 \frac{C_{I L} V_{I L}}{C_{a q} V_{a q}}=100 E F \varphi
$$

where $V_{\mathrm{IL}}$ and $V_{\text {aq }}$ are the volumes of the IL phase and the sample solution, respectively, and $\varphi$ is the phase ratio. Human breast milk samples were spiked with known amounts of NFX and BNZ solutions, so the final concentrations were $2.5,5.0,7.5,10.0$ and $15.0 \mu \mathrm{g} \mathrm{mL}^{-1}$. The concentrations of the target analytes in the extracts were within the linear range of the calibration curves. The EF was determined by spiking a measured amount of analyte in a known volume of human breast milk and then determining the final concentration extracted into the RTIL phase. The analyte was left in contact with the human breast milk matrix for $1 \mathrm{~h}$ before extraction. The $R \%$ was determined by measuring the initial volume of spiked human breast milk and the final volume of RTIL phase and using the EF parameter. The $R \%$ obtained for NFX at different concentration levels were higher than those for BNZ (Table 7), but close to the recoveries predicted by the RSM procedure (91.9 and $78.0 \%$, respectively). Figure 3 shows the typical chromatograms of the two antichagasic drugs before and after IL-DLLME, in a spiked human breast milk sample.

\subsection{Comparison of the proposed methodology with other procedures from literature}

No analytical techniques to determine NFX in human breast milk were found in the literature. Thus, a comparison with the figures of merit obtained in the present paper is not possible. For BNZ, the obtained results were compared with the ones obtained by Marson et al. [22]. They used a tandem microfiltration system after protein precipitation and HPLC-UV analysis, with no preconcentration step. They have calculated the LOD by using the current definition of IUPAC ( $3.3 S_{\mathrm{b}} / b$, where $S_{\mathrm{b}}$ is the intercept standard error and $b$ is the slope of the calibration curve). The obtained LOD was $0.3 \mu \mathrm{g}$ $\mathrm{mL}^{-1}$, LOQ $\left(9 S_{\mathrm{b}} / b\right)$ of $0.9 \mu \mathrm{g} \mathrm{mL} \mathrm{m}^{-1}$, a $\mathrm{R} \%$ of $70 \%$ with a precision lower than $10 \%$ and a linear range of 0.9 to $15 \mu \mathrm{g} \mathrm{mL} \mathrm{m}^{-1}$, intra-day accuracy of $6.6 \%$ and intra-day precision of $2.1 \%$. These LODs and 
LOQs cannot be compared with the ones obtained in the present study since the definitions used are different. However, the LODs and LOQs obtained here were calculated by a more rigorous procedure than that used in Ref. [22]. On the other hand, the $R \%(78 \%)$ was higher in this work, with a better precision (1.5\%) than that reported in Ref. [22]. Also, in this work, a wider linear range was obtained: from the LOQ (the value depends on the used procedure of IUPAC or $\mathrm{S} / \mathrm{N}$ ratio) to $29.16 \mu \mathrm{gL}^{-1}$ as compared with 0.9 to $15 \mu \mathrm{g} \mathrm{mL}^{-1}$ obtained by Marson et al. [22]. Finally, intra-day accuracy and intraday precision of $1.96 \%$ obtained in this work can be compared with 6.6 and $2.1 \%$ obtained in Ref. [22]. Thus, the recoveries and the upper LOQ were higher and the intra-day accuracy is lower in the present work.

\subsection{Analysis of BNZ and NFX in human breast milk}

As explained in Section 3.3, quantitative determinations were made by the standard addition method. Thus, linear regression of the peak areas vs. standard concentration at eight concentration levels made by triplicate (three different samples analyzed) were prepared. In Table 8 the antichagasic drug contents of human breast milk samples determined after the IL-DLLME-HPLCUV procedure are shown. For one sample of a patient treated with BNZ and for two samples of patients treated with NFX the drug was detected bellow the LOQ. For the other human breast milk samples, amounts of both drugs in the ppm range could be quantified precisely.

\section{$4 \quad$ Concluding remarks}

In this work, a simple and efficient method to quantify benznidazole and nifurtimox in human breast milk was developed. The methodology consists of a simple sample pre-treatment followed by an ionic liquid based dispersive liquid-liquid microextraction combined with LC and UV detection. For the extraction/preconcentration step, the ionic liquid 1-octyl-3-methylimidazolium hexafluorophosphate has been used as the "extraction solvent". A factorial design, called central 
composite design was used to find the optimum values for the significant variables affecting the extraction process: volume of ionic liquid, volume of disperser solvent, ionic strength and $\mathrm{pH}$. The $\mathrm{pH}$ and volume of ionic liquid were the most influential parameters affecting the extraction of benznidazole, while for nifurtimox, the volume of ionic liquid, the ionic strength and an interaction term (volume of ionic liquid $\mathrm{x} \mathrm{KCl}$ concentration) were significant.

The analytical technique is simple, fast, requires small amounts of extraction solvent, which is an environmentally friendly compound, and exhibits a wide range of linearity, very low limits of detection and quantification, excellent reproducibility intra- and inter-days and high recoveries for both antichagasic drugs.

\section{Acknowledgements}

Financial support from the Consejo Nacional de Investigaciones Científicas y Técnicas (CONICET), Agencia Nacional de Promoción Científica y Técnica (ANPCYT) and Facultad de Ciencias Exactas (UNLP) is gratefully acknowledged.

M. Reta and A. Califano are scientific members of CONICET and Professors of Analytical Chemistry and Experimental Design at the Universidad Nacional de La Plata (Argentina). J. M. Padró and R. N. Echevarría are researches fellows of CONICET, and R. Pellegrino Vidal is a research fellow of Comisión de Investigaciones Científicas de la Provincia de Buenos Aires (CIC-PBA), Argentina.

The authors also wish to thank to Prof. Dr. Guido Mastantonio and the student Ph.D. María E. Marsón from LaSeISiC-UNLP (CIC-PBA), La Plata, ARGENTINA, and the Drs. Jaime Altcheh and Facundo García-Bournissen from Hospital de Niños Ricardo Gutiérrez, Buenos Aires, ARGENTINA, for the human breast milk samples. 


\section{References}

[1] Dias, J. C. P., Silveira, A. C., Schofield, C. J., Mem. Inst. Oswaldo Cruz 2002, 97, 603-612.

[2] Rodriques Coura, J., de Castro, S. L., Mem. Inst. Oswaldo Cruz 2002, 97, 3-24.

[3] Teixeira, A. R., Nascimento, R. J., Sturm, N. R., Mem. Inst. Oswaldo Cruz 2006, 101, $463-$ 491.

[4] Jannin, J., Villa, L., Mem. Inst. Oswaldo Cruz 2007, 102, 95-97.

[5] Rassi, J. A., Rassi, A., Marin-Neto, J. A., Mem. Inst. Oswaldo Cruz 2009, 104, 152-158.

[6] Tanowitz, H. B., Kirchhoff, L. V., Simon, D., Morris, S. A., Weiss, L. M., Wittner, M., Clin. Microbiol. Rev. 1992, 5, 400-419.

[7] Rodríguez-Morales, A. J., Rev. Peru. Med. Exp. Salud Pública 2005, 22, 123-133.

[8] Sosa-Estani, S., Viotti, R., Segura, E. L., Mem. Inst. Oswaldo Cruz 2009, 104, 167-180.

[9] Boiani, M., Piacenza, L., Hernández, P., Boiani, L., Cerecetto, H., González, M., Denicola, A., Biochem. Pharmacol. 2010, 79, 1736-1745.

[10] Castro, J. A., Montalto de Mecca, M., Bartel, L. C., Rodríguez, C., Boletín ATA 2008, 22, 24 35.

[11] Castro, J. A., Diaz de Toranzo, E. G., Biomed. Environ. Sci. 1988, 1, 19-33.

[12] Castro, J. A., Biomed. Environ. Sci. 2000, 13, 271-279.

[13] Castro, J. A., Montalto de Mecca, M., Bartel, L. C., Hum. Exp. Toxicol. 2006, 25, 471-479.

[14] Rossi, D. T., Scott Wright, D., J. Pharm. Biomed. Anal. 1997, 15, 495-504.

[15] Kašparová, M., Plíšek, J., Solichová, D., Krčmová, L., Kučerová, B., Hronek, M., Solich, P., Talanta 2012, 93, 147-152.

[16] Scott, L. J., Perry, C. M., Drugs 2000, 60, 139-176.

[17] Gibson, T. P., Am. J. Med. 1996, 101, 47-53.

[18] Lee, C. R., McTavish, D., Sorkin, E. M., Drugs 1993, 46, 313-340.

[19] Lehmann, K. A., Drugs 1994, 47, 19-32.

[20] Padró, J. M., Marson, M. E., Mastrantonio, G. E., Altcheh, J., García-Bournissen, F., Reta, M., Talanta 2013, 107, 95-102. 
[21] Martínez, N., Marson, M. E., Mastrantonio, G. E., Raba, J., Cerutti, S., Talanta 2015, 131, 656-660.

[22] Marson, M. E., Padró, J. M., Reta, M. R., Altcheh, J., García-Bournissen, F., Mastrantonio, G., Ther. Drug Monit. 2013, 35, 522-526.

[23] Medenwald, H., Brandau, K., Schlossman, K., Arzneimittelforschung. 1972, 22, 1613-1617.

[24] Berijani, S., Assadi, Y., Anbia, M., Milani Hosseini, M.-R., Aghaee, E., J. Chromatogr. A 2006, 1123, 1-9.

[25] Rezaee, M., Assadi, Y., Milani Hosseini, M.-R., Aghaee, E., Ahmadi, F., Berijani, S., J. Chromatogr. A 2006, 1116, 1-9.

[26] Gathergood, N., Garcia, M. T., Scammells, P. J., Green Chem. 2004, 6, 166-175.

[27] Garcia, M. T., Gathergood, N., Scammells, P. J., Green Chem. 2005, 7, 9-14.

[28] Berthod, A., Ruiz-Ángel, M. J., Carda-Broch, S., J. Chromatogr. A 2008, 1184, 6-18.

[29] Acree, W. E., Grubbs, L. M., Encyclopedia of Analytical Chemistry, John Wiley \& Sons, Ltd., New York 2012.

[30] Liu, J.-F., Jiang, G.-B., Jönsson, J. A., TrAC, Trends Anal. Chem. 2005, 24, 20-27.

[31] Poole, C. F., J. Chromatogr. A 2004, 1037, 49-82.

[32] Cruz-Vera, M., Lucena, R., Cárdenas, S., Valcárcel, M., J. Chromatogr. A 2009, 1216, 64596465.

[33] Li, S., Gao, P., Zhang, J., Li, Y., Peng, B., Gao, H., Zhou, W., J. Sep. Sci. 2012, 35, 33893395.

[34] Liu, Y., Zhao, E., Zhu, W., Gao, H., Zhou, Z., J. Chromatogr. A 2009, 1216, 885-891.

[35] Ho, Y.-M., Tsoi, Y.-K., Leung, K. S.-Y., J. Sep. Sci. 2013, 36, 3791-3798.

[36] Zhang, L., Chen, F., Liu, S., Chen, B., Pan, C., J. Sep. Sci. 2012, 35, 2514-2519.

[37] Pena, M. T., Casais, M. C., Mejuto, M. C., Cela, R., J. Chromatogr. A 2009, 1216, 6356-6364.

[38] Garcia-Bournissen, F., Altcheh, J., Panchaud, A., Ito, S., Arch. Dis. Child. 2010, 95, 224-8.

[39] Montgomery, D. C., Design and Analysis of Experiments, John Wiley \& Sons, New York 1997.

[40] Box, G. E. P., Wilson, K. B., J. R. Stat. Soc. B 1951, 13, 1-45.

[41] Ferreira, S. L. C., dos Santos, W. N. L., Quintella, C. M., Neto, B. B., Bosque-Sendra, J. M., Talanta 2004, 63, 1061-1067. 
[42] Padró, J. M., Ponzinibbio, A., Agudelo Mesa, L. B., Reta, M., Anal. Bioanal. Chem. 2011, $399,2807-2820$.

[43] Mestorino, N., Marchetti, M. L., Turic, E., Pesoa, J., Errecalde, J., Anal. Chim. Acta 2009, 637, 33-39.

[44] Box, G. E. P., Draper, N. R., Empirical Model-Building and Response Surfaces, John Wiley \& Sons, New York 1987.

[45] Derringer, G., Suich, R., J. Qual. Technol. 1980, 12, 214-219.

[46] Massart, D. L., Vandeginste, B. G. M., Buydens, L. M. C., Dejong, S., Lewi, P. J., SmeyersVerbeke, J. Handbook of Chemometrics and Qualimetrics: Part A., Elsevier, Amsterdam 1997. 
Figure 2. Surface response plots of: a) percentage recovery of benznidazole ( $R \% \mathrm{BNZ})$ as a function of $\mathrm{pH}$ and volume of ionic liquid $\left(V_{\mathrm{IL}}\right)$, using $\mathrm{MeOH}$ volume $=93.84 \mu \mathrm{L}$ and $2.0(\%, \mathrm{w} / \mathrm{v}) \mathrm{KCl}$; b) percentage recovery of nifurtimox $(R \% \mathrm{NFX})$ as a function of $\mathrm{KCl}$ content $\left(C_{\mathrm{KCl}}\right)$ and $\mathrm{V}_{\mathrm{IL}},\left(V_{\mathrm{MeOH}}=\right.$ $84 \mu \mathrm{L}$ and $\mathrm{pH}=6$ ).
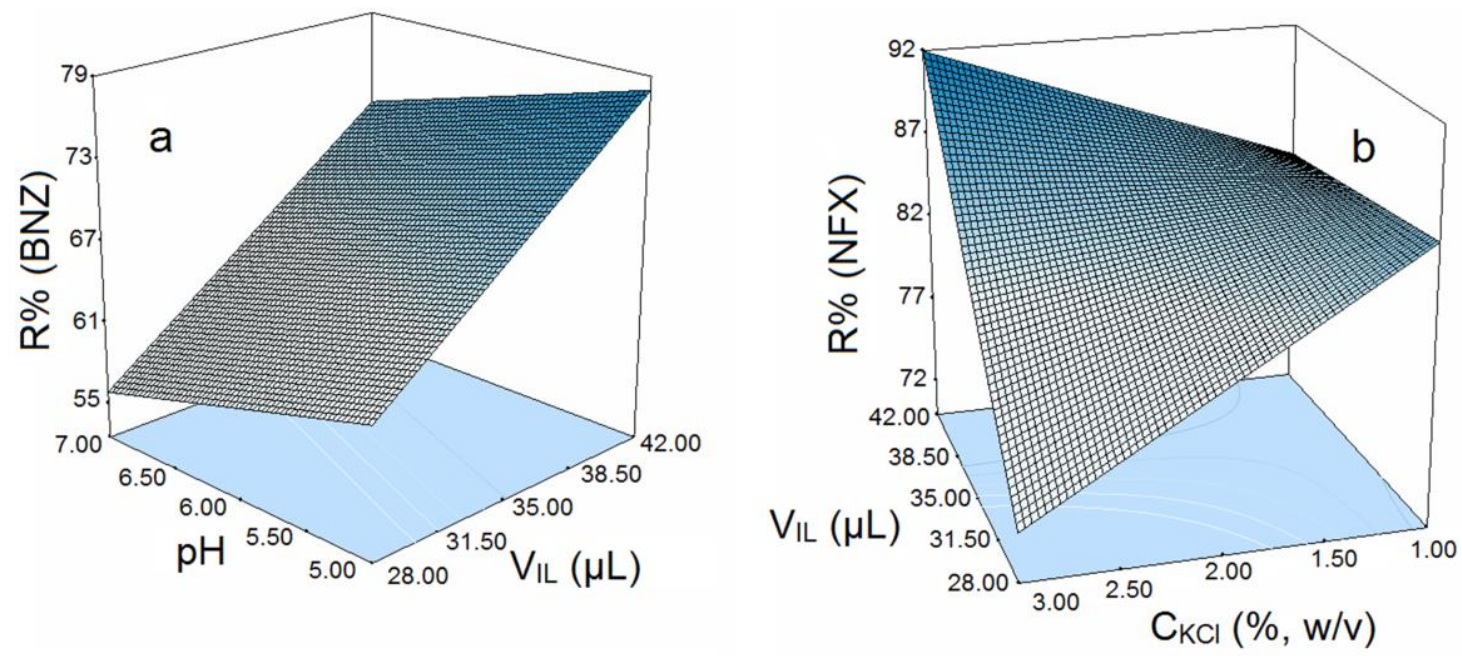
Figure 3. Chromatograms for protein-free human-breast milk sample spiked with BNZ (a) and NFX (b) at $2.5 \mu \mathrm{g} \mathrm{mL}{ }^{-1}$ before (dotted line) and after (solid line) IL-DLLME preconcentration.
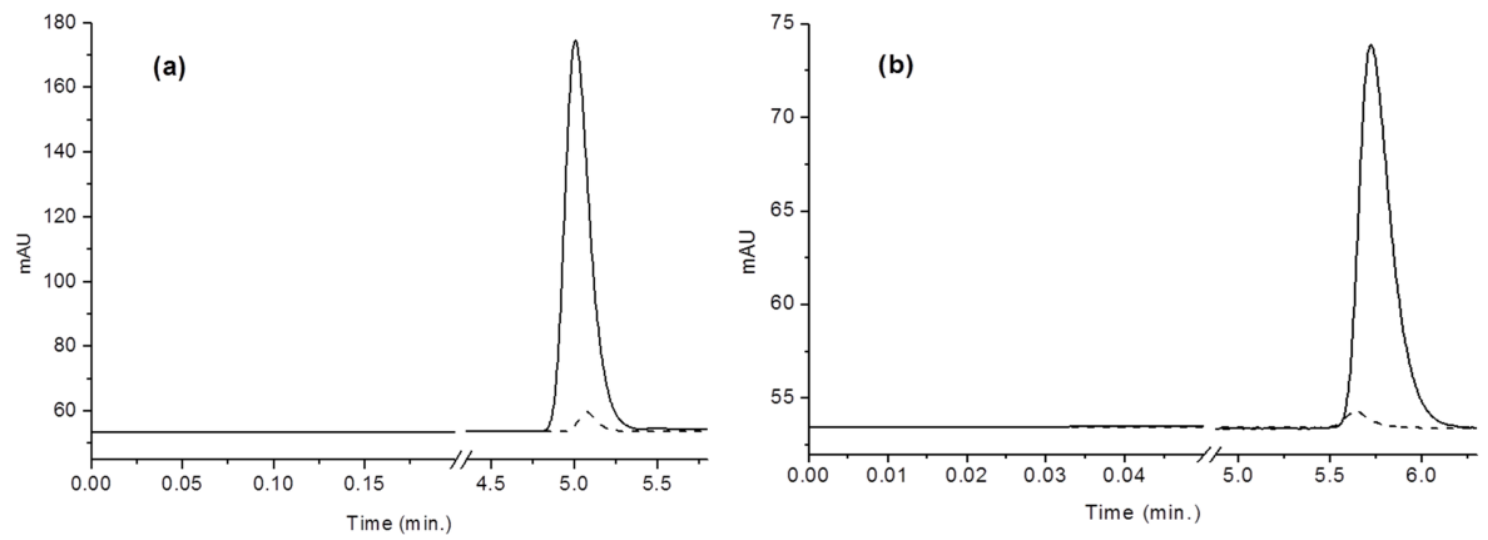
Table 1. Actual and codified factors: ionic liquid volumen $\left(\mathbf{V}_{\mathrm{LL}}\right)$, dispersant volumen $\left(\mathbf{V}_{\mathrm{MeOH}}\right)$, ionic strength ( $\mathrm{KCl}$ concentration, $\mathrm{C}_{\mathrm{Kcl}}$ ) and $\mathrm{pH}$ values according with the central composite design used.

\begin{tabular}{cccccc}
\hline & \multicolumn{5}{c}{ Codified factors } \\
\hline Actual Factors & $\mathbf{- 2}$ & $\mathbf{- 1}$ & $\mathbf{0}$ & $\mathbf{+ 1}$ & $\mathbf{+ 2}$ \\
\hline $\mathrm{V}_{\mathrm{IL}}(\mu \mathrm{L})$ & 28 & 31.5 & 35 & 38.5 & 42 \\
$\mathbf{V}_{\mathrm{MeOH}}(\mu \mathrm{L})$ & 56 & 70 & 84 & 98 & 112 \\
$\mathrm{C}_{\mathrm{KCl}}(\%, \mathrm{w} / \mathrm{v})$ & 1 & 1.5 & 2 & 2.5 & 3 \\
$\mathbf{p H}$ & 5 & 5.5 & 6.0 & 6.5 & 7 \\
\hline
\end{tabular}


Table 2. Comparison between the optimal values found for the considered variables for the ILDLLME obtained by the CCD and by the "step-by-step" [20] procedures.

\begin{tabular}{ccccc}
\hline & \multicolumn{5}{c}{ Factor } \\
\cline { 2 - 5 } Compound & $\mathrm{V}_{\mathrm{IL}}(\mu \mathrm{L})$ & $\mathrm{V}_{\mathrm{MeOH}}(\mu \mathrm{L})$ & $\mathrm{pH}$ & $\mathrm{C}_{\mathrm{KCI}}(\%, \mathrm{w} / \mathrm{v})$ \\
\hline BNZ & 42 & 101.2 & 5.00 & 1.98 \\
NFX & 42 & 80.0 & 5.91 & 2.97 \\
step-by-step $^{\mathrm{a})}$ & 35 & 84.0 & 6.12 & 2.00
\end{tabular}

a) optimal values, for both compounds in ultrapure water, found in our previous work [20]. 
Table 3. Calibration curves for NFX and BNZ after IL-DLLME of three samples without initial analyte content.

\begin{tabular}{cccc}
\hline Analyte & Linear regression & $\boldsymbol{r}^{\mathbf{2} \text { a }}$ & $\boldsymbol{N}^{\mathrm{b})}$ \\
\hline BNZ & & & \\
\hline Sample 1 & $y=(-24.0 \pm 8.2)+(3.5 \pm 0.2) x$ & 0.9724 & 24 \\
Sample 2 & $y=(-14.2 \pm 4.7)+(4.0 \pm 0.1) x$ & 0.9900 & 19 \\
Sample 3 & $y=(-12.2 \pm 4.3)+(4.42 \pm 0.08) x$ & 0.9969 & 19 \\
\hline NFX & & & \\
\hline Sample 1 & $y=(-38.1 \pm 16.1)+(4.5 \pm 0.4) x$ & 0.9327 & 22 \\
Sample 2 & $y=(-54.2 \pm 9.8)+(6.8 \pm 0.2) x$ & 0.9936 & 22 \\
Sample 3 & $y=(-121 \pm 18)+(7.9 \pm 0.3) x$ & 0.9929 & 22 \\
\hline
\end{tabular}

a) $r^{2}=$ determination coefficient.

b) $N=$ number of points. 
Table 4. $t$-Test at $5 \%$ probability for comparison of the calibration curves slopes obtained in Table 3.

\begin{tabular}{ccc}
\hline Comparison of slopes & $\boldsymbol{t}$ (calculated) & $\boldsymbol{t}^{\prime}$ (tabulated) \\
\hline $\boldsymbol{B N} \boldsymbol{Z}$ & & \\
Samples 1-2 & 2.020 & 2.010 \\
Samples 1-3 & 4.660 & 2.084 \\
Samples 2-3 & 2.843 & 2.109 \\
\hline $\boldsymbol{N F \boldsymbol { X }}$ & & \\
\hline Samples 1-2 & 5.374 & 2.090 \\
Samples 1-3 & 7.200 & 2.042 \\
Samples 2-3 & 3.464 & 2.042 \\
\hline
\end{tabular}


Table 5. Limits of detection (LOD) and quantification (LOQ) obtained for BNZ and NFX in human breast milk (concentration units in $\mu \mathrm{g} \mathrm{mL}^{-1}$ ).

\begin{tabular}{cccccc}
\hline Analyte & Linear range & LOD $^{\text {a) }}$ & LOD $^{\text {b) }}$ & LOQ $^{\text {c) }}$ & LOQ $^{\text {b) }}$ \\
\hline BNZ & LOQ-29.16 & 0.09 & 0.29 & 0.30 & 0.88 \\
NFX & LOQ-34.40 & 0.06 & 0.18 & 0.20 & 0.55 \\
\hline
\end{tabular}
a) $S / N=3$.
b) From calibration curve (actual IUPAC definition).
c) $\mathrm{S} / \mathrm{N}=10$. 
Table 6. Quantitative determinations of NFX and BNZ in spiked human breast milk, accuracy and reproducibility (concentrations in $\mu \mathrm{g} \mathrm{mL}^{-1}$ ).

\begin{tabular}{|c|c|c|c|c|}
\hline Analyte & $\boldsymbol{C}_{\text {added }}\left(\mu \mathrm{g} \mathrm{mL}^{-1}\right)$ & $\boldsymbol{C}_{\text {found }}\left(\mu \mathrm{g} \mathrm{mL}^{-1}\right)$ & Accuracy $(\%)^{a)}$ & Precision $\boldsymbol{R S D}(\%)^{\mathbf{b})}$ \\
\hline \multicolumn{5}{|l|}{ NFX } \\
\hline \multirow[t]{3}{*}{ Intra-day } & 5.0 & $4.8 \pm 0.3$ & -4.16 & 4.16 \\
\hline & 10.0 & $9.7 \pm 0.3$ & -3.09 & 1.55 \\
\hline & 15.0 & $14.5 \pm 0.2$ & -3.44 & 0.68 \\
\hline \multirow[t]{3}{*}{ Inter-day } & 5.0 & $5.2 \pm 0.5$ & 3.85 & 5.77 \\
\hline & 10.0 & $9.8 \pm 0.3$ & -2.04 & 2.04 \\
\hline & 15.0 & $14.8 \pm 0.4$ & -1.35 & 2.02 \\
\hline \multicolumn{5}{|l|}{ BNZ } \\
\hline \multirow[t]{3}{*}{ Intra-day } & 5.0 & $5.1 \pm 0.2$ & 1.96 & 1.96 \\
\hline & 10.0 & $9.8 \pm 0.3$ & -2.04 & 2.04 \\
\hline & 15.0 & $14.7 \pm 0.3$ & -2.04 & 1.36 \\
\hline \multirow[t]{3}{*}{ Inter-day } & 5.0 & $4.8 \pm 0.4$ & -4.16 & 6.25 \\
\hline & 10.0 & $9.7 \pm 0.4$ & -3.09 & 2.06 \\
\hline & 15.0 & $14.6 \pm 0.5$ & -2.73 & 2.05 \\
\hline
\end{tabular}

a) relative error percent; $E_{r}(\%)=\left(\left(C_{\text {added }}-C_{\text {found }}\right) / C_{\text {added }}\right) \times 100$.

b) $\mathrm{SD} / \overline{\mathrm{X}}_{\mathrm{av}} \times 100$. 
Table 7. Enrichment factors (EF), recoveries $(\boldsymbol{R} \%)$ and their $\mathbf{R S D} \%$ for human breast milk samples at different spiked levels of NFX and BNZ.

\begin{tabular}{cccccccc}
\hline & \multicolumn{3}{c}{$\mathrm{NFX}$} & & \multicolumn{3}{c}{ BNZ } \\
\cline { 2 - 4 } \cline { 6 - 8 } $\boldsymbol{C}\left(\mu \mathrm{g} \mathrm{mL}^{-1}\right)$ & $\boldsymbol{E F}$ & $\boldsymbol{R}(\%)^{\mathrm{a})}$ & $\boldsymbol{R S D}(\%)^{\mathrm{b})}$ & & $\boldsymbol{E F}$ & $\boldsymbol{R}(\%)$ & $\boldsymbol{R S D}(\%)^{\mathrm{b})}$ \\
\hline 2.5 & 33.8 & 90.0 & 1.2 & & 28.8 & 76.8 & 1.5 \\
5.0 & 33.5 & 89.3 & 0.8 & & 29.0 & 77.3 & 0.9 \\
7.5 & 33.0 & 90.8 & 0.8 & & 28.6 & 76.3 & 0.9 \\
10.0 & 33.0 & 90.6 & 1.0 & & 29.3 & 78.1 & 1.0 \\
15.0 & 32.8 & 87.8 & 0.7 & & 29.6 & 78.9 & 0.6 \\
$\mathrm{RSM}^{\mathrm{c})}$ & & 91.9 & & & 78.0 & \\
\hline
\end{tabular}

a) $R \%$ at $n=3$.

b) $\mathrm{SD} / \overline{\mathrm{X}}_{\mathrm{av}} \times 100$ calculated for recovery.

c) Predicted by the Response Surface Methodology. 
Table 8. Quantification of BNZ and NFX in eight human breast milk samples of different patients treated with each of the drugs. (samples were analized in triplicate).

\begin{tabular}{cccc}
\hline & BNZ & \multicolumn{2}{c}{ NFX } \\
\hline Sample & $\boldsymbol{C}\left(\mu \mathrm{g} \mathrm{mL}^{-1}\right)$ & Sample & $\boldsymbol{C}\left(\mu \mathrm{g} \mathrm{mL}^{-1}\right)$ \\
\hline Sample 1 & $7.1 \pm 0.3$ & Sample 1 & $<\mathrm{LOQ}$ \\
Sample 2 & $5.2 \pm 0.4$ & Sample 2 & $8.2 \pm 0.3$ \\
Sample 3 & $3.1 \pm 0.3$ & Sample 3 & $8.1 \pm 0.4$ \\
Sample 4 & $<$ LOQ & Sample 4 & $<\mathrm{LOQ}$ \\
\hline
\end{tabular}

